\title{
Voice recognition impairment in a blind Capgras patient
}

\author{
I. Reid' 1 A.W. Young ${ }^{2}$ and D.J. Hellawell ${ }^{3}$ \\ 'Department of Mental Health, University of Aberdeen Medical School, Foresterhill, \\ Aberdeen AB9 2ZD, ${ }^{2}$ Medical Research Council Applied Psychology Unit, \\ 15 Chaucer Road, Cambridge CB2 2EF and ${ }^{3}$ Department of Clinical Neurosciences, \\ Western General Hospital, Crewe Road, Edinburgh EH4 2XU, UK
}

Correspondence to: I. Reid or A. Young at above addresses

\begin{abstract}
We report a case of a blind woman, M.N., who experienced the Capgras delusion. She thought that her pet cat had been replaced by a replica which was "ill-intentioned" towards her. M.N.'s case shows that the basis of the Capgras delusion cannot lie exclusively in damage to the visual system. However, testing of M.N.'s auditory recognition abilities revealed a deficit in the recognition of familiar voices. This impairment is consistent with the view that the Capgras delusion may arise in connection with damage to recognition mechanisms, and parallels findings of face processing impairments in sighted Capgras patients.
\end{abstract}

Keywords: Capgras delusion - Cognitive neuropsychiatry - Voice recognition

\section{INTRODUCTION}

The Capgras delusion involves the belief that familiar people have been replaced by "dummies" or impostors. Enthusiasm for psychodynamic accounts of this curious phenomenon has been tempered by observations of a clear organic contribution in the form of demonstrable brain injury in some cases (Joseph, 1986; Lewis, 1987).

Lewis (1987), Anderson (1988), and Ellis and Young (1990) have independently suggested that the basis of the Capgras delusion may lie in damage to neuroanatomical pathways responsible for appropriate emotional reactions to familiar visual stimuli, so that "a correct perception fails to evoke the normal affective memories tied to it" (Lewis, 1987 , p. 120). This idea has been further developed into the proposal that the Capgras delusion represents the patient's attempt to make sense of the fact that visual stimuli no longer produce appropriate affective responses, and that the genesis of this delusion therefore involves an interaction of impairments at two levels (Wright et al., 1993; Young et al., 1993). One set of contributory factors involve anomalous perceptual experience, and the other factors lead to an incorrect interpretation of this, with misattributions due to suspiciousness playing an important role.

We report here a case of a blind woman, M.N., who experienced the Capgras delusion. This case shows that the basis of the Capgras delusion cannot lie exclusively in damage to the visual system. However, testing of M.N.'s voice recognition abilities revealed a deficit which paral- lels findings of face processing impairments in sighted Capgras patients (Lewis, 1987; Ellis and Young, 1990).

\section{CASE REPORT}

M.N. was a 32 year old single woman who lived alone. She was congenitally blind, suffering from retinal aplasia, but able to look after herself independently, having held a number of office jobs. At the time of presentation, however, she had become increasingly reclusive and had given up work. She had made her way to a local hospital complaining that her life was threatened by neighbours who were "brainwashing" her by pumping gas into her house. At interview she expressed a number of persecutory delusions, which by a neighbour's account had been present for approximately a year. She was also experiencing auditory hallucinations, taking the form of voices referring to her in the third person. She believed that her movements were under her neighbours' control.

Of particular interest, M.N. felt that her pet cat (who had been her sole companion for 10 years) had been replaced by a "bad" duplicate, which was "cruel" and "ill-intentioned" towards her. Initially, she described the cat as somehow different in its manner and behaviour, and later reported that the cat appeared to make different sounds from usual, and that its fur had changed. The exact nature of these subtle differences could not be specified further by M.N. There was only one duplicate of the cat, and 
questioning did not reveal any complaints of duplication of anything or anyone else.

M.N. was otherwise in good health, and routine physical investigations were normal. An MRI scan performed shortly after admission showed only a small area of abnormality in the left corona radiata, considered to be either a small infarct or an artefact of no significance.

A diagnosis of paranoid schizophrenia was made, and M.N. was treated initially with oral neuroleptic medication, and subsequently with a regular depot preparation. Her delusions and hallucinations gradually resolved, but she remained withdrawn and poorly motivated to care for herself. She abandoned her beliefs about her cat, and came to accept that it had not changed. After 2 months in hospital she was able to return to her home, under regular supervision and with the provision of a home help. To date, she has had no recurrence of her psychotic symptoms, but remains isolated and poorly motivated.

\section{INVESTIGATION OF M.N.'S RECOGNITION ABILITIES}

M.N.'s performance of auditory recognition and voice processing tasks was investigated some 3 months after her discharge from hospital (while still taking neuroleptic medication), using a series of tasks constructed in order to parallel those used in the investigation of face processing impairments in sighted patients suffering delusional misidentification (Young et al., 1990). Stereognosis (the ability to recognize objects by touch) was also tested, and found to be both quick and accurate using either left or right hand (15/15 correct left hand; $15 / 15$ correct right hand).

Auditory recognition and voice processing tasks given to M.N. included:

(1) Identification of environmental sounds: an audio tape of 20 clips of everyday sounds (rain, telephone, etc.), lasting $8 \mathrm{~s}$ each. M.N. was asked to identify each sound.

(2) Recognition of voices and names: an audio tape was prepared, which included clips of 30 voices of familiar (famous) people and 10 unfamiliar people, lasting approximately $5 \mathrm{~s}$ each. The clips were chosen so that the content of each was relatively clichéd, and as far as possible did not give direct clues to the speaker's identity, which could only be inferred from the voice itself. Familiar and unfamiliar voices were presented in an unpredictable order. M.N. was asked whether or not each voice was familiar, and if so to identify the person by occupation or name. Because of its importance to our investigation, this voice recognition test was given twice, in separate sessions two months apart.

A parallel task was also given, using the names of the 30 familiar people who had been used in the voice recognition test, and 10 unfamiliar names. These names were read to
M.N. one at a time in a pseudo-random order, and she was asked whether or not each name was familiar, and if so to identify the person by occupation.

(3) Unfamiliar voice matching: 40 pairs of sentences were presented, with the second sentence in each pair following the first after a $2 \mathrm{~s}$ interval. The two sentences in each pair were always different, but half ( 20 pairs) were spoken by the same person, and half (20 pairs) by two different people of the same sex. M.N. was asked to decide whether each pair of sentences was spoken by the same or different people.

(4) Recognition memory: M.N.'s recognition memory for voices was tested by presenting a series of taped sentences, which were read twice each by four different unfamiliar voices. This was followed by pairs of voices reading each sentence, one of which was the voice in the initial phase. M.N. was asked to identify this member of each pair. The procèdure was repeated five times, using different sets of voices, to give a score out of 20 possible correct responses for recognition memory for voices. The reason for testing the voices in blocks of four at a time was that pilot work showed the task to be otherwise excessively difficult even for normal controls.

A test of recognition memory for words suitable for a blind person was also derived as closely as possible from the procedure used in Warrington's (1984) Recognition Memory Test (which uses visual presentation). The 50 words from the Recognition Memory Test were read out at the rate of one every $3 \mathrm{~s}$ for a "pleasant or unpleasant" decision to ensure the subject's orientation to the task (thus copying Warrington's procedure, but with auditory instead of visual presentation), and then recognition memory was tested by reading pairs of words consisting of one word from the original list paired with a distractor, with M.N. having to choose which member of each pair had been in the original list.

(5) Vocal expression: three separate tasks were given. The first (labelling of tone of voice) used 24 neutral sentences spoken with one of three possible emotional expressions (happy, sad, angry). M.N. was asked to say whether each sentence was spoken in a happy, sad, or angry way. The second task (labelling of sentence content) involved 24 sentences with happy, sad, or angry emotional content, spoken with neutral (flat) intonation. M.N. was asked whether the sentence had happy, sad or angry content. The third task (prosody control task) involved 24 neutral sentences spoken with an intonation which implied a question, statement, or exclamation. M.N. was asked to determine whether each was a question, statement, or exclamation.

Results for these various auditory and voice processing tasks are summarized in Table I, which also gives details of the performance of six blind but otherwise healthy control subjects of mean age 27 years for several tasks (mean 
TABLE I. Performance of M.N. on auditory recognition and voice processing tasks, with means, standard deviations, and ranges of scores for blind control subjects, and means and standard deviations of scores for sighted control subjects

\begin{tabular}{|c|c|c|c|c|c|c|}
\hline & \multirow[t]{2}{*}{ M.N. } & \multicolumn{3}{|c|}{$\begin{array}{l}\text { Blind controls } \\
\quad(n=6)\end{array}$} & \multicolumn{2}{|c|}{$\begin{array}{l}\text { Sighted controls } \\
\qquad(n=20)\end{array}$} \\
\hline & & Mean & S.D. & Range & Mean & S.D. \\
\hline Identification of environmental sounds & $15 / 20 \dagger$ & 17.17 & 1.72 & $15-19$ & 18.00 & 1.34 \\
\hline \multicolumn{7}{|l|}{ Identification of voices and names } \\
\hline \multirow{2}{*}{\multicolumn{7}{|c|}{$\begin{array}{l}\text { Famous voices } \\
\text { Recognized as familiar }\end{array}$}} \\
\hline & \multicolumn{6}{|c|}{ Recognized as familiar } \\
\hline First session & $8 / 30 \#^{\star \star} \ddagger$ & 22.25 & 5.56 & $16-24$ & 21.70 & 5.67 \\
\hline Retest & $7 / 30 \#^{\star \star} \ddagger$ & & & & & \\
\hline \multicolumn{7}{|l|}{ Correctly identified } \\
\hline First session & $6 / 30 \# \dagger$ & 16.25 & 6.88 & $8-27$ & 18.15 & 5.64 \\
\hline Retest & $5 / 30 \# * \ddagger$ & & & & & \\
\hline \multicolumn{7}{|l|}{ Unfamiliar voices } \\
\hline Correct rejections & $10 / 10$ & 8.75 & 1.26 & $7-10$ & 9.20 & 1.01 \\
\hline \multicolumn{7}{|l|}{ Famous names } \\
\hline Recognized as familiar & $29 / 30$ & & & & & \\
\hline Correctly identified & $24 / 30$ & & & & & \\
\hline \multicolumn{7}{|l|}{ Unfamiliar names } \\
\hline Correct rejections & $9 / 10$ & & & & & \\
\hline Unfamiliar voice matching & $38 / 40$ & & & & 39.05 & 1.10 \\
\hline \multicolumn{7}{|l|}{ Recognition memory } \\
\hline Voices & $19 / 20$ & 19.50 & 0.84 & $18-20$ & 17.80 & 1.64 \\
\hline Words & $41 / 50$ & 43.83 & 3.54 & $40-49$ & & \\
\hline \multicolumn{7}{|l|}{ Vocal expressions } \\
\hline Labelling tone of voice & $23 / 24$ & 22.33 & 1.03 & $21-23$ & 22.35 & 1.18 \\
\hline Labelling content & $23 / 24$ & 23.50 & 0.55 & $23-24$ & 23.80 & 0.52 \\
\hline Prosody control task & $22 / 24$ & 22.00 & 1.79 & $19-24$ & 20.80 & 2.35 \\
\hline
\end{tabular}

Scores marked \# are outside the range for blind control subjects; scores significantly below the mean for blind controls are marked as follows: ${ }^{*} z>1.65, p<0.05,{ }^{* \star} z>2.33, p<0.01$; scores significantly below the mean for sighted controls are marked as follows: $\nmid z>1.65, p<0.05, \ddagger z>2.33, p<0.01$.

number correct, standard deviation, and range of scores), and means and standard deviations for 20 sighted control subjects aged 20-39 years (mean age 29.5 years, S.D. $=5.82$ ).

M.N.'s ability to recognize environmental sounds $(15 / 20$ correct) fell at the bottom of the normal range for the blind controls, and was slightly poorer than that of sighted controls. As well as this poor recognition of environmental sounds, there was a severe impairment in the recognition of familiar voices.

The voice recognition test was given twice, in separate sessions 2 months apart. On both occasions M.N. was markedly poor at recognizing familiar voices as familiar $(8 / 30$ correct in the first test, $7 / 30$ on retest; both scores were significantly below and outside the range of the blind control subjects, and significantly poorer than sighted controls). She was also poor at identifying the famous voices $(6 / 30$ correctly identified by occupation or name in the first test, $5 / 30$ on retest; both scores were outside the range of the blind control subjects, and significantly poorer than sighted controls). However, the parallel test with famous names showed that M.N.'s problem in recognizing famous voices was not due to lack of familiarity with these people, since she recognized $29 / 30$ of their names as familiar, and could provide appropriate semantic information to $24 / 30$. Hence her problem did seem to be one of voice recognition perse.

The data given in Table I also show that M.N. had no detectable problem in matching unfamiliar voices, in recognition memory for voices or words, or in the processing of prosody and vocal expressions of emotion. Her impairment was therefore specific to auditory recognition tasks involving familiar stimuli, and did not reflect any generalized defect of auditory information processing; it therefore seems unlikely that the problem was a side effect of medication. 


\section{DISCUSSION}

To the best of our knowledge, the only other published reports of the Capgras delusion in blind patients are those of Signer et al. (1990) and Rojo et al. (1991). In neither case was there any attempt to investigate voice recognition abilities. It is worth noting that sensory deprivation generally may predispose to paranoid symptomatology, although blind subjects are much less likely to be so affected than the deaf (Mayer-Gross et al., 1969). The specific auditory recognition deficits reported here have not been implicated in the development of paranoid psychoses, although the hard of hearing are over-represented among samples of patients suffering from paranoid psychoses in later life (Cooper, 1976).

The existence of the Capgras delusion in blind people has important implications for the idea that the basis of the delusion lies in damage to neuroanatomical pathways responsible for appropriate emotional reactions to familiar visual stimuli (Anderson, 1988; Ellis and Young, 1990; Lewis, 1987). On this view, the finding is at first sight contrary to what might be expected, as Rojo et al. (1991) correctly point out. However, there are two possible modifications of the hypothesis which could accommodate our observation. It is possible that whilst the Capgras delusion may reflect a delusional interpretation of loss of appropriate emotional reactions to visual stimuli in many cases, there is a parallel form of the delusion affecting the auditory modality, in which duplicates are postulated on the basis of loss of appropriate emotional reactions to auditory stimuli. Alternatively, the Capgras delusion might be linked to a more central (i.e. polysensory) loss of emotional significance of familiar stimuli regardless of the modality of input. In this case, it might be predicted that sighted Capgras patients may also exhibit deficits in voice recognition.

Whether or not either of these modifications is correct, M.N.'s case shows that the basis of the Capgras delusion cannot lie exclusively in damage to the visual system. However, testing of M.N.'s auditory recognition abilities revealed a deficit of voice recognition which was present even when she no longer experienced the delusion. This impairment is consistent with the view that the Capgras delusion may arise in connection with damage to the recognition system, and parallels findings of face processing impairments in sighted Capgras patients (see Lewis, 1987; Ellis and Young, 1990; Young et al., 1993). It will therefore be of interest to know whether equivalent impairments of voice recognition are found for other blind Capgras patients.

An additional unusual feature of M.N.'s case was that the delusion did not affect another person, but her pet cat. However, M.N. lived alone and without close contact with relatives, so that the cat was probably of particular import- ance to her. Although it is more usual for close relatives to be the object of the Capgras delusion, the literature does contain at least one other case involving a pet cat (Wallis, 1986).

Neuro-imaging showed only a small area of abnormality involving the left cerebral hemisphere, but the finding of impaired auditory recognition is more consistent with some form of right hemisphere or perhaps bilateral dysfunction, since Van Lancker and Canter (1982) found that voice recognition impairments are usually associated with right hemisphere damage. Other studies have reported either bilateral or primarily right hemisphere abnormalities in Capgras cases (Förstl et al., 1991; Joseph, 1986).

\section{Acknowledgement}

We gratefully acknowledge the support provided by a grant from the EJLB Foundation, Canada.

\section{REFERENCES}

Anderson DN (1988) The delusion of inanimate doubles. British Journal of Psychiatry, 153, 694-699.

Cooper AF (1976) Deafness and psychiatric illness. British Journal of Psychiatry, 129, 216-226.

Ellis HD and Young AW (1990) Accounting for delusional misidentifications. British Journal of Psychiatry, 157, 239-248.

Förstl H, Burns A, Jacoby R and Levy R (1991) Neuroanatomical correlates of clinical misidentification and misperception in senile dementia of the Alzheimer type. Journal of Clinical Psychiatry, 52, 268-271.

Joseph AB (1986) Focal central nervous system abnormalities in patients with misidentification syndromes. Bibliotheca Psychiatrica, 164, 68-79.

Lewis SW (1987) Brain imaging in a case of Capgras' syndrome. British Journal of Psychiatry, 150, 117-121.

Mayer-Gross W, Slater E and Roth M (1969) Clinical Psychiatry. Baillière Tindall and Cassell, London.

Rojo VI, Caballero L, Iruela LM and Baca E (1991) Capgras' syndrome in a blind patient. American Journal of Psychiatry, 148, 1272.

Signer SF, Van Ness PC and Davis RJ (1990) Capgras's syndrome associated with sensory loss. Western Journal of Medicine, 152, 719-720.

Van Lancker DR and Canter GJ (1982) Impairment of voice and face recognition in patients with hemispheric damage. Brain and Cognition, 1, 185-195.

Wallis G (1986) Nature of the misidentified in the Capgras syndrome. Bibliotheca Psychiatrica, 164, 40-48.

Warrington EK (1984) Recognition Memory Test. NFER-Nelson, Windsor.

Wright S, Young AW and Hellawell DJ (1993) Sequential Cotard and Capgras delusions. British Journal of Clinical Psychology, 32, 345-349.

Young AW, Ellis HD, Szulecka TK and de Pauw KW (1990) Face processing impairments and delusional misidentification. Behavioural Neurology, 3, 153-168.

Young AW, Reid I, Wright S and Hellawell DJ (1993) Face-processing impairments and the Capgras delusion. British Journal of Psychiatry, 162, 695-698.

(Received 14 September 1993; accepted 30 October 1993) 


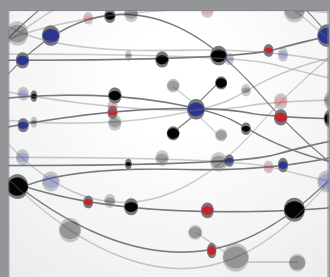

The Scientific World Journal
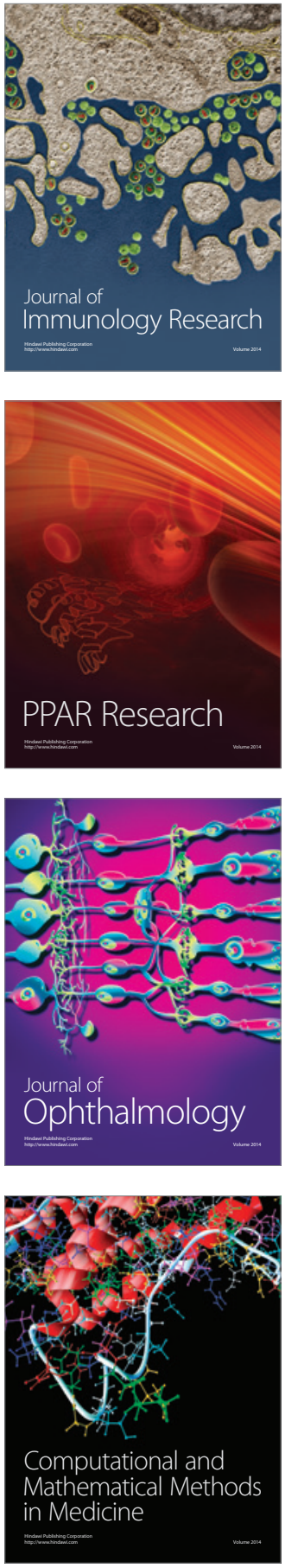

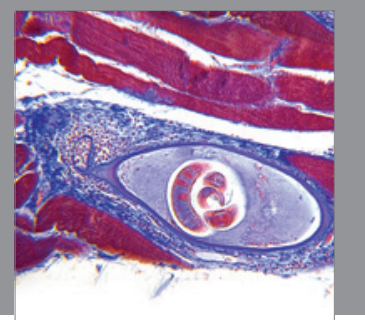

Gastroenterology

Research and Practice
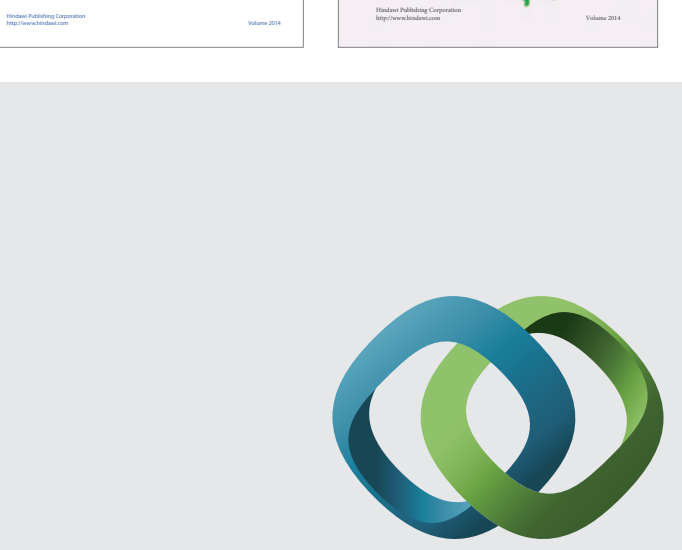

\section{Hindawi}

Submit your manuscripts at

http://www.hindawi.com
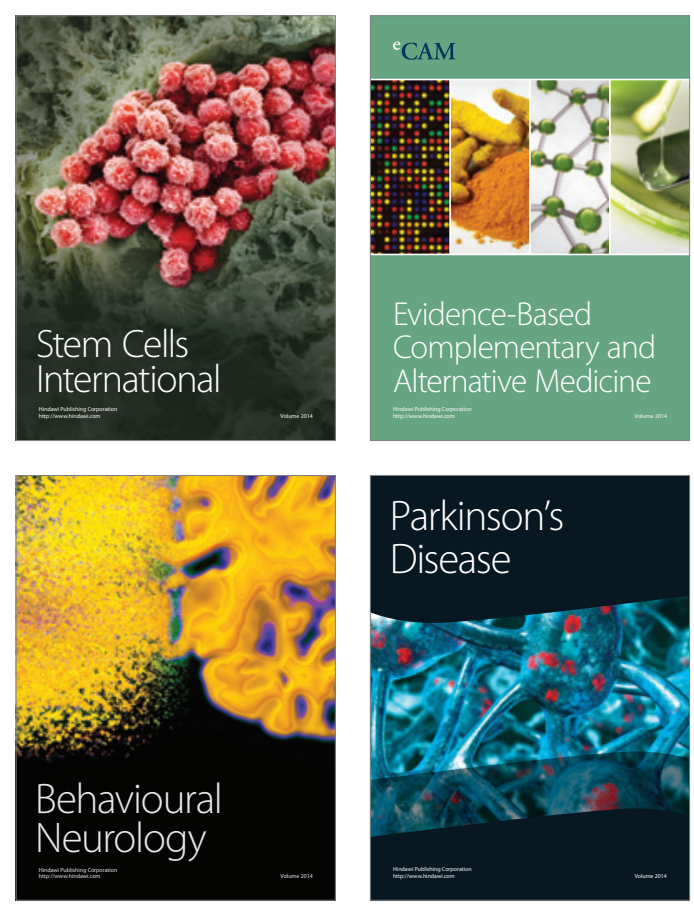

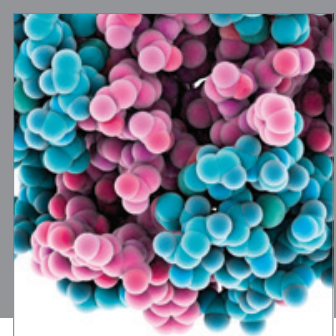

Journal of
Diabetes Research

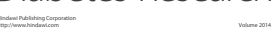

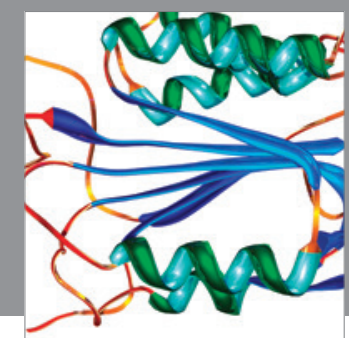

Disease Markers
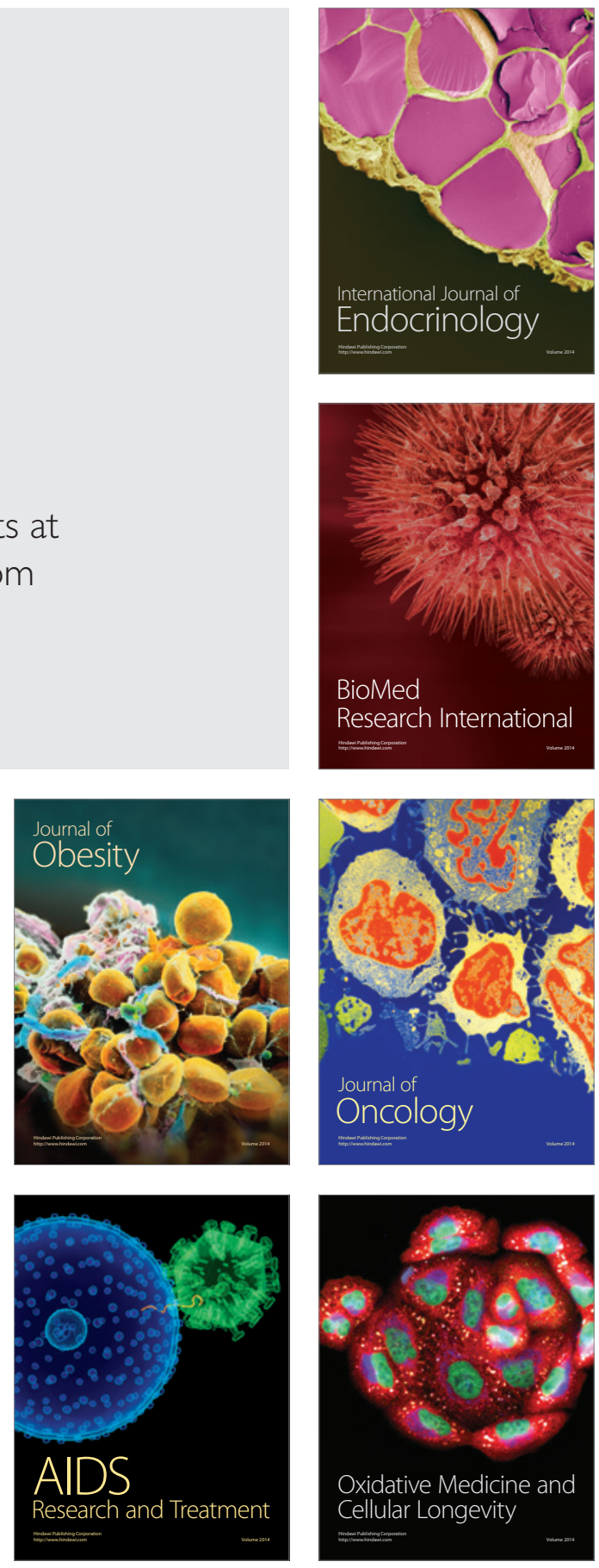